\section{9 "MITRACLIP, A SUMMIT WELL WORTH REACHING FOR". OUTCOME OF TRANSCATHETER MITRAL VALVE CLIP FOR THE MANAGEMENT OF MITRAL REGURGITATION IN HIGH RISK GROUP PATIENTS UNSUITABLE FOR SURGICAL INTERVENTION}

doi:10.1136/heartjnl-2012-301877b.79

F Keshavarzi, ${ }^{*}$ A Hoye, A L Clark, R Oliver, J G Cleland, F Alamgir. Castle Hill Hospital, East Yorkshire, UK

Introduction Mitral regurgitation (MR) can cause or complicate heart failure, affecting patient quality of life and survival. Surgical mitral valve (MV) repair or replacement is effective but associated with substantial morbidity and mortality in high-risk groups. MV clipping is a potential alternative, minimally invasive and lower risk procedure that has been shown to have similar benefits to surgical repair in randomised trials. The aim of this study was to assess the impact of the MitraClip procedure on MR and functional class in clinical practice in patients who were refused MV surgery.

Method Patient demographics including age, gender, cardiac / valve structure and function, aetiology of $M R$, duration of the procedure, complications, duration of hospital stay and the NYHA class pre and post procedure were recorded. SPSS statistical tool was used to analyse the data.

Results 30 patients unsuitable for surgery were referred to our centre. Twelve patients were excluded because of co-morbidities and were considered clinically inappropriate MitraClip $(n=5 ; 42 \%)$ and/ or because of unsuitable valve morphology ( $n=7 ; 58 \%)$. Of 18 patients who underwent the procedure, the mean age was 73 (range 58-85) years and 14 were men. MR was functional in 15 (83\%), due to valve prolapse in 2 and degenerative valve disease in one. Two clips were deployed in four patients. The median procedure time was $227 \mathrm{~min}$ (123-380 min). Procedures longer than $2 \mathrm{~h}$ involved multiple clip application as well as coronary angioplasty. Mean hospital stay was 6 days (1-18 days) and $78 \%$ of patients were discharged within 1 week. Complications included failure to remove the catheter after the MitraClip application in one patient with severe pre-existing heart failure who later died of heart failure, bleeding from the groin and septicaemia. One patient who had been hospitalised for 5 months with severe heart failure died from pericardial tamponade most likely due to atrial wall damage. Among surviving patients, MR severity improved to grade I/II in $84 \%$, to grade III in $16 \%$. Of patients initially in NYHA IV, one died, one improved to NYHA I, one to NYHA III and one did not improve. Of 14 patients initially in NYHA III, Nine (62\%) improved to NYHA I/ II, one died and four did not improve substantially.

Conclusion In high-risk patients who have been declined mitral valve surgery, MV repair using the MitraClip procedure can be done with a moderate risk and with a high procedural success rate. Advances in expertise and technology will improve efficacy and patient safety.

\section{SAFETY OF LMWH FOR BRIDGING ANTICOAGULATION BEFORE FULL WARFARINISATION IN MECHANICAL VALVES}

doi:10.1136/heartjnl-2012-301877b.80

S G Jones, ${ }^{*} O$ Valencia, E E J Smith, M Shannon, M Jahangiri. St. George's Hospital, London, UK

Objective Early following mechanical heart valve replacement, patients are at high risk of valve thrombosis and bleeding complications. There are no published guidelines on the use of specific bridging therapies until full warfarinisation is achieved. Use of unfractionated heparin (UFH) in these patients is widespread. We set out to examine the rate of thrombosis shortly after surgery until full anticoagulation was achieved in patients receiving low molecular weight heparin (LMWH).

Methods Prospectively collected data on 765 patients who had mechanical aortic or mitral valve replacement from January 2002 to September 2011 was reviewed. Of these patients 182 received intravenous or subcutaneous UFH due to co-morbidities and were excluded from our analysis. The remaining patients received 5000 units of Dalteparin (Pfizer) twice daily from the first postoperative day. Post operative echocardiography results were examined for valve thrombosis, and evidence of gastrointestinal or intracranial bleeds was sought. Anticoagulation was deemed therapeutic when the INR was $>2.0$.

Results 583 patients who had mechanical aortic or mitral valve replacement at our unit from January 2002 to September 2011 were included. These consisted of 451 aortic and 173 mitral valves. 59 patients had multiple valves replaced, including concomitant tricuspid replacement. 385 (66\%) patients were male, the mean age was 56 years. There were 4 in-hospital deaths $(0.69 \%)$. Mean time to reach therapeutic INR was 3.4 days. 18 (3.1\%) patients had resternotomy for bleeding. No patients had evidence of valve thrombosis. There were no intracranial bleeds.

Conclusion Use of LMWH compares favourably with published series of bridging with UFH. There was a low incidence of postoperative bleeding complications and no evidence of valve thrombosis. Use of standardised dose of LMWH simplifies administration and there is no need for monitoring of the activated partial thromboplastin time.

\section{ACUTE DERANGEMENT OF CARDIAC ENERGY METABOLISM AND OXYGENATION DURING STRESS IN HYPERTROPHIC CARDIOMYOPATHY -A POTENTIAL MECHANISM FOR SUDDEN CARDIAC DEATH}

doi:10.1136/heartjnl-2012-301877b.81

S Dass, * J Suttie, T Karamitsos, H Watkins, S Neubauer. University of Oxford, Oxford, UK

Introduction Hypertrophic cardiomyopathy (HCM) is the commonest cause of sudden cardiac death in the young. The sarcomere mutations increase the energy cost of contraction and impaired resting energetics (phosphocreatine/adenosine triphosphate, PCr/ATP, measured by 31Phosphorus MR Spectroscopy, 31P MRS) has been shown. In addition, abnormal perfusion in HCM is an independent predictor of clinical deterioration and death. However, whether microvascular dysfunction is severe enough to result in deoxygenation has not been explored. We hypothesise: (1) Cardiac energetics are further impaired acutely during exercise in HCM, but not in normals or athletes (physiological hypertrophy); (2) This energetic abnormality contributes to diastolic impairment, is independent of the degree of hypertrophy or patchy fibrosis; (3) Tissue oxygenation during stress is impaired in HCM.

Methods 31P MRS (3T) was performed in 35 age and gender matched HCM patients, 12 athletes and 20 normal controls at rest and during $8 \mathrm{~min}$ of steady leg exercise lying prone with $2.5 \mathrm{~kg}$ weights. BOLD signal intensity change (SIÄ) and myocardial perfusion reserve index (MPRI) were measured with adenosine stress.

Results There was no difference in resting PCr/ATP between normals $(2.14 \pm 0.36)$ and athletes $(2.04 \pm 0.32, \mathrm{p}=0.36)$. Resting $\mathrm{PCr} /$ ATP was significantly reduced in HCM $(1.71 \pm 0.35, p<0.0001)$. During exercise there was a further reduction in PCr/ATP in HCM $(1.56 \pm 0.31, \mathrm{p}<0.05)$ but not in normals $(2.13 \pm 0.34, \mathrm{p}=0.98)$, or athletes $(2.09 \pm 0.50, \mathrm{p}=0.63)$. There was no correlation between cardiac mass, average wall thickness and rest or exercise energetics. Peak filling rates (PFR) were significantly reduced in HCM (rest: 